\title{
Rational Beliefs as Cognitive Protective Factors Against Posttraumatic Stress Symptoms
}

\author{
Philip Hyland • Rebecca Maguire • Mark Shevlin • \\ Daniel Boduszek
}

Published online: 15 August 2014

(C) Springer Science+Business Media New York 2014

\begin{abstract}
The current study investigated the predictions of rational emotive behaviour therapy's (REBT) model of psychological health that rational beliefs would act as cognitive protective factors against posttraumatic stress responses. The study aimed to contribute original data regarding the role of functional cognitions in the prevention of posttraumatic stress symptomology. A model consistent with REBT's theory of psychological health was investigated through the use of structural equation modelling among a sample of 309 international emergency service personnel who had all been exposed to a significant life trauma. Results indicated that the REBT model of posttraumatic stress responses was found to be an acceptable fit of the data $\left(\chi^{2}=199.99, d f=94, p<.001\right.$; RMSEA $=.06$ (CI $90 \%=.05 / .08) ; \mathrm{SRMR}=.05 ; \mathrm{CFI}=.93 ; \mathrm{TLI}=.91)$ and explained $76 \%$ of variance in posttraumatic stress symptoms. Self-acceptance beliefs and high frustration tolerance beliefs negatively predicted posttraumatic stress responses; noncatastrophizing beliefs positively predicted posttraumatic stress responses; and indirect effects were observed between preference beliefs and posttraumatic stress responses via self-acceptance and high frustration tolerance beliefs. Results suggest that rational beliefs are negatively associated with posttraumatic stress responses and support the REBT model of psychological health. Current results contribute important information regarding the role of functional cognitions in the alleviation of posttraumatic stress symptoms.
\end{abstract}

P. Hyland $(\bowtie) \cdot$ R. Maguire

School of Business, National College of Ireland, Mayor Street, IFSC, Dublin, Ireland e-mail: Philip.hyland@ncirl.ie

M. Shevlin

School of Psychology, University of Ulster, Londonderry, UK

D. Boduszek

Department of Behavioural and Social Sciences, University of Huddersfield, Huddersfield, UK 
Keywords Rational emotive behaviour therapy (REBT) - Rational beliefs · Posttraumatic stress disorder (PTSD) - Structural equation modelling (SEM)

\section{Introduction}

Social-cognitive models of posttraumatic stress disorder (PTSD) focus on the effect that experience of a traumatic life event has on an individual's existing belief system (e.g., Ehlers and Clark 2000; Resick and Schnicke 1993). Contemporary cognitive-behavioural theories of PTSD are predicated upon the principle that dysfunctional cognitive processing of a traumatic event leads to the development of a range of negative and distorted belief systems about the self, others, the world, the future, and the traumatic event itself (Clark and Beck 2010). The presence of these belief systems is hypothesised to exert a negative impact on a number of cognitive processes leading to faulty trauma memories and attentional cognitive biases towards threatening stimuli. These processes consequently lead to the characteristic intrusive and hyperarousal symptoms which are subsequently appraised in a negative manner leading to maladaptive behavioural control strategies which involve avoidance and emotion control/suppression efforts (see Clark and Beck 2010).

Research efforts have predominately focused on identifying the role of dysfunctional thoughts and irrational beliefs in the development of posttraumatic stress symptoms. Naturally, a range of psychometrically validated measures of posttraumatic stress cognitions are widely utilized such as the Posttraumatic Cognitions Inventory (Foa et al. 1999), the Personal Beliefs and Reactions Scale (Resick et al. 1991), and the Posttraumatic Maladaptive Belief Scale (Vogt et al. 2012). While these scales are highly informative in terms of identifying cognitive vulnerability factors for the development and/or maintenance of posttraumatic stress responses, comparatively less is known about the types of functional cognitions that may protect against the development of such psychopathology.

The majority of cognitive-behavioural models of PTSD (e.g. Ehlers and Clark 2000; Clark and Beck 2010) and measures of posttraumatic cognitions (Foa et al. 1999; Resick et al. 1991; Vogt et al. 2012) derive directly from the theoretical foundations of Beck's Cognitive Therapy (CT) model of psychopathology. Beck's CT model is just one of many cognitive-behavioural models of psychopathology (David and Szentagotai 2006). It is important that researchers interested in developing comprehensive cognitive-behavioural therapy (CBT) models of PTSD do not neglect incorporating the different types of dysfunctional cognitions outlined in other CBT models. An alternative and highly influential CBT model of psychopathology is Ellis' rational emotive behaviour therapy (REBT: see David and Szentagotai 2006; Hyland and Boduszek 2012 for a full discussion on the similarities and differences of CT and REBT theory).

The REBT model of psychopathology is built around Ellis' (e.g., 1994) ABC model, where irrational belief processes (B) are viewed as important etiopathogenetic variables in the development of maladaptive cognitive-emotional-behavioural- 
psychophysiological symptoms (C) that can arise following exposure to a life adversity (A). Specifically, demandingness beliefs are conceptualised as the primary irrational belief process in the development of psychopathology and their impact of various psychopathological outcomes are mediated via a set of secondary irrational beliefs (catastrophizing, low frustration tolerance, and depreciation beliefs). A good deal of empirical evidence exists within the scientific literature to support the general REBT model of psychopathology and the interrelations between the irrational beliefs (e.g., David et al. 2002; DiLorenzo et al. 2007; Hyland et al. 2014a).

Recently a series of studies have provided empirical support for the theoretical predictions of REBT's model of psychopathology in the context of posttraumatic stress responses. Using structural equation modelling procedures, Hyland et al. (2014a) demonstrated that a model consistent with contemporary REBT theory of psychopathology provided a good fit of the data, and that theses irrational belief explained $67 \%$ of variance in intrusive symptoms; $50 \%$ of variance in avoidance symptoms; $67 \%$ of variance in dysphoria symptoms; and $56 \%$ of variance in hyperarousal symptoms, respectively. Subsequent studies have supported the role that irrational beliefs play in posttraumatic stress symptomology (Hyland et al., in press), as well as the role of trauma-specific variations of each irrational belief type in the prediction of reporting clinically relevant posttraumatic stress symptoms (Hyland et al. 2013). These results provide support for the REBT model of psychopathology, and highlight the role of irrational beliefs as cognitive vulnerability factors in posttraumatic stress responses. However, in addition to identifying important cognitive vulnerability factors, REBT theory is also well suited to identifying certain cognitive protective factors that may serve to mitigate the development of posttraumatic stress responses.

REBT theory also specifies a model of psychological health. This model too is structured around Ellis' ABC theory of psychological disturbance. According to the REBT model of psychological health, rational beliefs (B) are viewed as important sanogenetic factors in the development of maladaptive cognitive-emotionalbehavioural-psychophysiological symptoms (C) following exposure to a life adversity (A). In other words, responding to a life adversity with a set of rational belief is hypothesised to protect an individual from developing maladaptive psychological responses (DiLorenzo et al. 2011). Within the REBT model of psychological health, preference beliefs (flexible desires or wishes) are viewed as the primary rational belief process, and the impact of preference beliefs on various psychological outcomes is predicted to be mediated via a set of secondary rational belief process. These secondary rational belief processes include: (i) non-catastrophizing beliefs, in which a person rates adverse life events in a realistically negative manner; (ii) high frustration tolerance beliefs, in which a person accurately assesses their own ability to withstand and tolerate the distress of experiencing an adversity; and (iii) acceptance beliefs, in which a person adopts an unconditionally accepting view of themselves, others, and the world when things do not occur as they wish, and rates only aspects of their own behaviour (or that of others or their environment) rather than rating their whole self (or another or their environment). 
Rational beliefs hold a central place in REBT theory given that the clinical practice of REBT is focused on disputing irrational beliefs and fostering rational belief alternatives in order to alleviate psychopathology (Ellis and Dryden 2007). Despite this, little empirical work has been conducted to evaluate the role of rational beliefs in protecting against the development of psychopathology; the organisation of the rational belief processes; or whether rational beliefs are negatively associated with posttraumatic stress responses following exposure to a traumatic life event. Considerable evidence has been accrued from exploratory factor analysis (Bernard 1998), confirmatory factor analysis (Fulop 2007; Macavei 2002; see Hyland et al. 2014b), longitudinal research (DiLorenzo et al. 2011), and neuroscientific research (Tiba 2003) that rational and irrational beliefs represent distinct cognitive processes. It is therefore imperative that researchers investigate the independent role of rational beliefs in both functional and dysfunctional outcomes.

Recent research findings have begun to provide initial evidence that rational beliefs may play a protective role against the emergence and/or maintenance of posttraumatic stress responses. In a study utilizing sequential moderated multiple regression analysis, Hyland et al. (2014c) demonstrated that rational beliefs can moderate the impact of irrational beliefs on posttraumatic stress symptoms. Specifically, the authors demonstrated that increased levels of self-acceptance beliefs served to significantly reduce the impact of catastrophizing beliefs on levels of posttraumatic stress symptomology. Additionally, high frustration tolerance beliefs, and self-acceptance beliefs were both negatively (and weakly) associated with posttraumatic stress levels.

The general lack of evidence relating to the hypothesised protective role of rational beliefs in the development and/or maintenance of psychopathology is a major problem for REBT theory. Moreover, no previous studies could be identified which have sought to assess the hypothesised organisation of the rational belief processes. The current study was performed in order to redress this deficiency in the REBT literature, and to substantially contribute to the wider PTSD literature in a number of ways.

First, we sought to test the predictions of REBT's model of psychological health within a sample of emergency service workers who had all been exposed to at least one traumatic life event. The lack of evidence in relation to the validity of the REBT model of psychological health precluded the ability to make a definitive a priori hypothesis as to how well the REBT model would fit the obtained data, and how much of the variance in posttraumatic stress responses would be explained, therefore this research aim was largely exploratory in nature.

Second, we sought to investigate the hypothesised interrelations between the rational beliefs and their proposed direct and indirect effects on posttraumatic stress symptomology. Based on clear theoretical predictions (David et al. 2010), we hypothesised that positive direct effects would be observed between preference beliefs and non-catastrophizing, high frustration tolerance, and acceptance beliefs, respectively; that negative direct effects would be observed between noncatastrophizing, high frustration tolerance, and self-acceptance beliefs, respectively, and posttraumatic stress responses; and finally that negative indirect effects would 
be observed from preference beliefs to posttraumatic stress responses via noncatastrophizing, high frustration tolerance, and acceptance beliefs, respectively.

\section{Method}

\section{Participants}

An opportunistic sample of $365(\mathrm{~N}=365)$ international emergency service personnel were recruited from active-duty or medical leave to participate in the current study. For the purposes of the current study 309 participants were included $(84.66 \%)$. Fifty-six individuals were excluded from the analyses due to refusal to complete the questionnaire. The sample included 210 males $(68 \%)$ and 99 females (32\%) and these individuals ranged in age from 23 to 65, with a mean age of 38.16 years $(\mathrm{SD}=8.73$ ). All participants included in the current study had reported experiencing at least one significant life trauma. The most commonly reported traumatic event was being involved in a serious accident $(60.4 \%, \mathrm{n}=189)$, followed by a non-sexual assault by a stranger $(56.9 \%, \mathrm{n}=178)$, and military combat $(42.5 \%, \mathrm{n}=133)$. Ninety participants reported their trauma taking place more than 5 years ago (29\%), 68 reported that the trauma occurred 3-5 years ago (22\%), 87 reported the trauma occurring between 6 months and 3 years previously (28\%), 40 reported the trauma occurring between 3 and 6 months previously (13\%), 11 reported the trauma occurring between 1 and 3 months previously (4\%), and 13 individuals reported that the trauma occurred less than 1 month previous (4\%). The majority of the participants resided in suburban areas $(44 \%, \mathrm{n}=137)$, and urban areas $(37 \%, 115)$, and the remainder indicated that they resided in rural areas $(19 \%, \mathrm{n}=57)$. The majority of the sample possessed at least a secondary/ high school level diploma $(56 \%, \mathrm{n}=173)$ while 106 held a bachelor's degree (34\%), and 30 participants possessed a master's degree or better (10\%). The majority of respondents were currently married $(\mathrm{n}=153,50 \%)$, while $19 \%$ reported their marital status to be single $(\mathrm{n}=60), 21 \%$ were cohabiting with a partner $(n=65)$, and $10 \%$ were divorced $(n=31)$.

\section{Procedures}

Participants were informed of the nature of the study being undertaken either by a member of the research team or a superior officer in their organisation. Each participant's involvement in the research project was voluntary, and no obligations were placed upon potential respondents nor were any inducements employed to recruit the sample. Each participant was assured about confidentiality and those who chose to take part in the research project had the option of completing either an anonymous self-administered paper-and-pencil version of the questionnaire or an electronic version which was completed and returned via email. The majority of respondents chose the paper-and-pencil option $(63.11 \%, \mathrm{n}=195)$. No relationship existed between the research team and the participants in the study. 


\section{Materials}

The Posttraumatic Stress Diagnostic Scale (PDS: Foa et al. 1997) is a 49-item selfreport measure of the severity of posttraumatic stress symptomology related to a particular traumatic event. The PDS assess all aspects of a PTSD diagnosis from Criteria A to F as outlined in the Diagnostic and Statistical Manual of Mental Disorders IV (DSM-IV: American Psychiatric Association [APA], 1994). The PDS measures the nature of the traumatic experience, the duration of the experienced symptoms, the impact of the experienced symptoms on daily functioning, and the severity of the symptoms. Seventeen items measure each of the identified symptoms of PTSD along a four-point Likert scale. Respondents rate the severity of each symptom from a score of 0 ("not at all or only one time") to 3 ("5 or more times a week/almost always"). This produces a total range of scores from 0 to 51 with higher scores indicating higher levels of posttraumatic stress symptomology. Scores from 0 to 10 reflect mild symptoms of PTSD; scores from 11 to 20 reflect moderate symptoms of PTSD; scores from 21 to 35 reflect moderate-to-severe symptoms of PTSD; while scores from 36 to 51 reflect severe symptoms of PTSD. The PDS possess strong psychometric properties with Griffin, Uhlmansiek, Resick, and Mechanic (2004) demonstrating that it shares a high correlation $(r=.71)$ with the Clinician-Administered PTSD scale (Blake et al. 1998). Cronbach alpha levels for the PDS are reported in Table 1.

The Abbreviated Version of the Attitudes and Belief Scale 2 (AV-ABS2: Hyland et al. 2014b) is a 24-item self-report measure of rational and irrational beliefs, as defined by current REBT theory (David et al. 2010). The AV-ABS2 measures all four irrational belief processes (demandingness, catastrophizing, low frustration tolerance, and self-depreciation beliefs) and all four rational belief processes (preferences, non-catastrophizing, high frustration tolerance, and self-acceptance beliefs). Each subscale is measured via three items and all items are scored along a five-point Likert scale from 1 ("Strongly Disagree") to 5 ("Strongly Agree"). Possible scores of each subscale range from 3 to 15, with higher scores in each case indicating higher levels of the respective belief process. The construct validity of the AV-ABS2 has been demonstrated in a previous confirmatory factor analytic study (Hyland et al. 2014b), and its psychometric properties were demonstrated to be superior to the full length Attitudes and Beliefs Scale-2 (DiGiuseppe et al. 1988).

For the purposes of the current study we were only concerned with the 12 items measuring the four rational belief processes. Items of the AV-ABS2 include, "I want to do well at important tasks, but I realize that I don't have to do well at these important tasks just because I want to." (Preferences): "It is unfortunate when I am frustrated by hassles in my life, but I realize it's only disappointing and not awful to experience hassles." (Non-Catastrophizing): "It's only frustrating not doing well at some tasks, but I know I can stand the frustration of performing less than well." (High Frustration Tolerance): and "When people whom I want to like me disapprove of me, I know I am still a worthwhile person" (Self-Acceptance). Each rational belief subscale of the AV-ABS2 exhibited acceptable internal consistency with Cronbach's Alpha levels all above .60 (see Pallant 2007). 
Table 1 Descriptive statistics and reliability of all variables

\begin{tabular}{lrrrll}
\hline & Mean & \multicolumn{1}{c}{ SD } & Range & Possible range & Cronbach's Alpha \\
\hline Posttraumatic stress & 11.36 & 10.76 & $0-41$ & $0-51$ & .94 \\
Preferences & 9.57 & 1.92 & $3-15$ & $3-15$ & .74 \\
Non-catastrophizing & 11.60 & 2.60 & $3-15$ & $3-15$ & .61 \\
High frustration tolerance & 10.55 & 2.86 & $3-15$ & $3-15$ & .62 \\
Self-acceptance & 11.64 & 4.09 & $3-15$ & $3-15$ & .95 \\
\hline
\end{tabular}

Analysis

Descriptive statistics and preliminary analysis were conducted within Statistical Package for the Social Sciences (SPSS) 21. The theoretical model was analysed using structural equation modelling (SEM) techniques. SEM is a combination of two analytical procedures; confirmatory factor analysis (CFA) which assesses the measurement component of a theoretical model, and path analysis which assesses the relationship between latent variables. Within an SEM framework, the structural and measurement elements of analysis are estimated simultaneously (MacCallum and Austin 2000). A number of other features make the use of SEM procedures appropriate for the current analysis. These include controlling for systematic and random measurement error and the ability to simultaneous test for both direct and indirect effects within a model (Bollen 1989). The SEM analysis was conducted in Mplus version 7.0 (Muthen and Muthen 2012) with Robust Maximum Likelihood (MLR) estimation.

The overall fit of the model was assessed using a range of goodness-of-fit statistics and assessment of the appropriateness of the model parameters. Kline's (2005) suggestions for determination of good model fit were followed for the SEM analyses; a Chi square-to-degrees of freedom $\left(\chi^{2}: d f\right)$ ratio less than $3: 1$; Comparative Fit Index (CFI; Bentler 1990) and Tucker Lewis Index (TLI; Tucker and Lewis 1973) values greater than .90; root-mean-square error of approximation (RMSEA: Steiger 1990) and standardized root-mean-square residual (SRMR: Joreskog and Sorbom 1981) values of .05 or less indicates good model fit, while values less than .08 suggest acceptable model fit.

\section{Results}

\section{Descriptive Statistics}

The mean level of posttraumatic stress symptoms for the entire sample was 11.36 $(\mathrm{SD}=10.76$; scores ranged from 0 to 41 ) indicating that the sample on average exhibited low-to-moderate levels of posttraumatic stress symptoms, however the large SD value indicates that there is large variation around this value. The mean scores for each rational belief process were moderate-to-high (see Table 1 for details). 


\section{Measurement Models}

Based on extensive findings regarding the factor structure of posttraumatic stress indicators (e.g., Yufik and Simms 2010), three alternative models of the PDS (Foa et al. 1997) were investigated. Model 1 is a four-factor solution (Intrusions, Avoidance, Emotional Numbing, and Hyperarousal) first suggested by King et al. (1998); Model 2 is an alternative four-factor solution (Intrusions, Avoidance, Dysphoria, Hyperarousal) first suggested by Simms et al. (2002); and Model 3 is the DSM-IV-TR's three-factorial solution. The Simms et al. 'Dysphoria' model was found to be the best fitting model yielding the most impressive fit statistics $\left(\chi^{2}=152.94, \quad d f=113, \quad p<.001 ; \quad\right.$ CFI $=.98 ; \quad$ TLI $=.98, \quad$ RMSEA $=.03 ;$ SRMR $=.03)$. These four subscales were consequently used as measured variables within the full structural model in order to construct a posttraumatic stress (PTS) latent variable.

\section{Structural Model}

The REBT rational beliefs model of posttraumatic stress symptoms (Fig. 1) was thus developed and included five latent variables: (i) preference beliefs (P), (ii) noncatastrophizing beliefs (NC), (iii) high frustration tolerance beliefs (HFT), and (iv) self-acceptance beliefs (SA), all measured via three items of the AV-ABS2, along with (v) PTS measured via intrusions, avoidance, dysphoria, and hyperarousal. Factor loadings for each observed variable on their respective latent variable were all statistically significant $(p<.001)$, positive, and greater than 0.40 thus satisfying the recommendations of Hair et al. (1998) for satisfactory factor loadings (see Table 2 for full details).

The REBT rational beliefs model of posttraumatic stress responses indicated in Fig. 1 was the final model settled upon following one post hoc modification which involved the inclusion of a correlated error variance between avoidance and hyperarousal. The model initially tested that included no correlated error variance produced unsatisfactory fit statistics $\left(\chi^{2}=263.90, \quad d f=95, \quad p<.001\right.$; RMSEA $=.08($ CI $90 \%=.07 / .09)$; SRMR $=.06$; CFI $=.90 ;$ TLI $=.87)$. Modification indices indicated that model fit would be substantially improved with the inclusion of the aforementioned correlated error. Following this addition to the model, the model was found to be a satisfactory fit of the data $\left(\chi^{2}=199.99\right.$, $d f=94, p<.001 ; \mathrm{RMSEA}=.06$ (CI $90 \%=.05 / .08) ; \mathrm{SRMR}=.05 ; \mathrm{CFI}=.93$; TLI $=.91)$. The model accounted for $76 \%$ of variance in posttraumatic stress symptoms.

Table 3 displays the standardized and unstandardized (direct and indirect) regression weights for the specified REBT rational beliefs model of posttraumatic stress responses. Preference beliefs had a weak-to-moderate positive, direct effect on non-catastrophizing beliefs $(\beta=.38, p<.001)$; a strong positive, direct effect on high frustration tolerance beliefs $(\beta=.86, p<.001)$; and a moderately-strong, positive direct effect on self-acceptance beliefs $(\beta=.56, p<.001)$. All three secondary rational belief processes were found to significantly predict levels of posttraumatic stress. Self-acceptance beliefs were the strongest predictor, with 


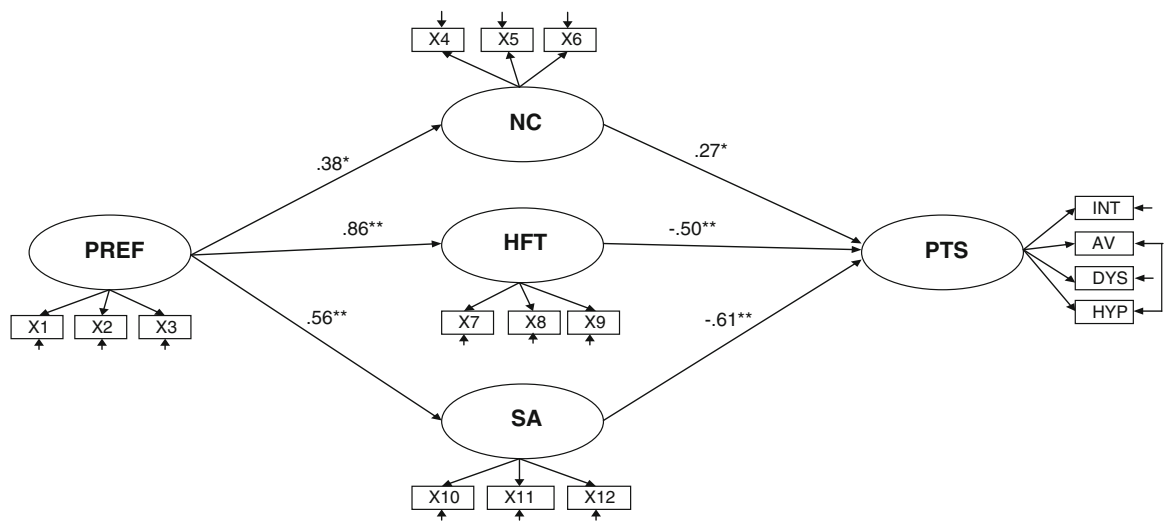

Fig. 1 REBT psychological health model of posttraumatic stress symptoms. PREF preference, $N C$ noncatastrophizing, HFT high frustration tolerance, $S A$ self-acceptance, PTSD posttraumatic stress disorder symptoms, $X 1-X 12$ Rational belief indicators of the AV-ABS2; INT Intrusions, $A V$ Avoidance, DYS dysphoria, HYP hyperarousal. Statistical significance: $* * p<.001,{ }^{*} p<.01$

results indicating a moderate-to-strong, negative direct relationship with posttraumatic stress responses $(\beta=-.61, p<.001)$. A moderate, negative direct effect was also observed between high frustration tolerance beliefs and levels of posttraumatic stress $(\beta=-.50, p<.001)$. These results demonstrate that higher levels of selfacceptance and higher levels of high frustration tolerance predict decreased levels of posttraumatic stress symptoms. Interestingly a positive, weak, direct effect was observed between non-catastrophizing beliefs and posttraumatic stress responses $(\beta=.27, p=.002)$. This result suggests that increased levels of this rational belief are weakly associated with increased levels of posttraumatic stress symptomology.

Two statistically significant, negative, indirect effects were also observed between preference beliefs and levels of posttraumatic stress symptomology. The strongest indirect relationship was observed between preference beliefs and posttraumatic stress symptoms via high frustration tolerance beliefs $(\beta=-.43$, $p=.001$ ), followed by preference beliefs and posttraumatic stress symptoms via self-acceptance beliefs $(\beta=-.34, p<.001)$. No indirect effect was identified between preference beliefs and posttraumatic stress symptoms via non-catastrophizing beliefs $(\beta=.10, p=.06)$.

\section{Discussion}

The current study was carried out with two primary objectives in mind. First, we sought to provide the first empirical investigation of REBT's model of psychological health in the prediction of posttraumatic stress responses, and secondly, we sought to investigate the predictions of REBT theory regarding the interrelations of the rational beliefs. This study was carried out in order to contribute important and original data to the wider trauma literature regarding the types of functional cognitions that can prevent or alleviate posttraumatic stress symptoms. 
Table 2 Standardized and unstandardized factor loadings (SE)

\begin{tabular}{|c|c|c|c|}
\hline Item & $\beta$ & $\mathrm{B}$ & $\mathrm{SE}$ \\
\hline \multicolumn{4}{|l|}{ PTSD } \\
\hline Intrusions & .91 & 1.00 & - \\
\hline Avoidance & .56 & .37 & .04 \\
\hline Dysphoria & .87 & 1.56 & .09 \\
\hline Hyperarousal & .56 & .46 & .03 \\
\hline \multicolumn{4}{|l|}{ Preferences } \\
\hline $\begin{array}{l}\text { I do not want to fail at important tasks but I realize that I } \\
\text { do not have to perform well just because I want to }\end{array}$ & .66 & 1.00 & - \\
\hline $\begin{array}{l}\text { I want to perform well at some things, but I do not have to } \\
\text { do well just because I want to }\end{array}$ & .68 & .96 & .10 \\
\hline $\begin{array}{l}\text { I want to do well at important tasks, but I realize that I } \\
\text { don't have to do well at these important tasks just } \\
\text { because I want to }\end{array}$ & .71 & 1.13 & .15 \\
\hline \multicolumn{4}{|l|}{ Non-catastrophizing } \\
\hline $\begin{array}{l}\text { It is unfortunate when I am frustrated by hassles in my } \\
\text { life, but I realize it's only disappointing and not awful to } \\
\text { experience hassles }\end{array}$ & 60 & 1.00 & - \\
\hline $\begin{array}{l}\text { When life is hard and I feel uncomfortable, I realize it is } \\
\text { not awful to feel uncomfortable or tense, only } \\
\text { unfortunate and I can keep going }\end{array}$ & .58 & 1.03 & .22 \\
\hline $\begin{array}{l}\text { It's bad to be disliked by certain people, but I realize it is } \\
\text { only unfortunate to be disliked by them }\end{array}$ & .57 & 1.00 & .17 \\
\hline \multicolumn{4}{|l|}{ High frustration tolerance } \\
\hline $\begin{array}{l}\text { I do not like to be uncomfortable, tense or nervous, but I } \\
\text { can tolerate being tense }\end{array}$ & .58 & 1.00 & - \\
\hline $\begin{array}{l}\text { I get distressed if I'm not doing well at important tasks, } \\
\text { but I can stand the distress of failing at important tasks }\end{array}$ & .43 & .69 & .14 \\
\hline $\begin{array}{l}\text { It's only frustrating not doing well at some tasks, but I } \\
\text { know I can stand the frustration of performing less than } \\
\text { well }\end{array}$ & .81 & 1.20 & .13 \\
\hline \multicolumn{4}{|l|}{ Self-acceptance } \\
\hline $\begin{array}{l}\text { When people whom I want to like me disapprove of me, I } \\
\text { know I am still a worthwhile person }\end{array}$ & .91 & 1.00 & - \\
\hline $\begin{array}{l}\text { Even when my life is tough and difficult, I realize that I } \\
\text { am a person who is just as good as anyone else even } \\
\text { though I have hassles }\end{array}$ & .98 & 1.11 & .03 \\
\hline $\begin{array}{l}\text { When my life becomes uncomfortable, I realize that I am } \\
\text { still a good person even though I am uncomfortable }\end{array}$ & .90 & .98 & .04 \\
\hline
\end{tabular}

All factor loadings are statistically significant $(p<.001)$

With regards to the first objective, results of the SEM analysis provided support for the REBT model of psychological health. The hypothesised model was found to provide acceptable fit of the data across all fit indices, following the inclusion of a correlated error between avoidance and hyperarousal. The presence of this correlated error between two of the indicators of posttraumatic stress symptomology 
Table 3 Standardized and unstandardized regression weights (with SE) for the REBT-based structural equation model of posttraumatic stress symptoms

\begin{tabular}{llll}
\hline Variables & $\beta$ & $\mathrm{B}$ & $\mathrm{SE}$ \\
\hline Direct influence & & & \\
Preference $\Longrightarrow$ Non-catastrophizing & $.38^{*}$ & .31 & .13 \\
Preference $\Longrightarrow$ High frustration tolerance & $.86^{* *}$ & .82 & .13 \\
Preference $\Longrightarrow$ Self-acceptance & $.56^{* *}$ & .88 & .14 \\
Non-catastrophizing $\Longrightarrow$ PTS & $.27^{*}$ & 1.23 & .43 \\
High frustration tolerance $\Longrightarrow$ PTS & $-.50^{* *}$ & -1.91 & .38 \\
Self-acceptance $\Longrightarrow$ PTS & $-.61^{* *}$ & -1.41 & .19 \\
Indirect influence & & & .21 \\
Preference $\Longrightarrow$ PTS via non-catastrophizing & .1 & .38 & .38 \\
Preference $\Longrightarrow$ PTS via high frustration tolerance & $-.43^{* *}$ & -1.57 & .26 \\
Preference $\Longrightarrow$ PTS via self-acceptance & $-.34^{* *}$ & -1.24 & \\
$R^{2}$ & & & \\
PTS $\mathrm{R}^{2}=.76$, SE $=.05, p<.001$ & & & \\
Fit indices & & & \\
$\chi^{2}=199.99, d f=94, p<.001 ;$ RMSEA $=.06$ (CI $\left.90 \%=.05 / .08\right) ;$ SRMR $=.05 ;$ CFI $=.93 ;$ \\
TLI $=.91$
\end{tabular}

Statistical significance: $* p<.01 ; * * p<.001$

is likely due to the use of a non-clinical sample, and suggestive of the possible presence of some non-specific distress factor. The $\chi^{2}$-to-df ratio for the final REBT model was approximately $2: 1$ indicating good fitting model, according to Klein's (2005) recommendations. Furthermore, the SRMR value also suggested a good fitting model. The RMSEA, CFI, and TLI values suggested an acceptable fitting model. In addition to providing an adequate fit of the data, the model was capable of explaining an impressive percentage of variance $(76 \%)$ in levels of posttraumatic stress symptoms among the current sample. Although the inclusion of a correlated error variance is not ideal, when results are considered in their entirety they suggest that the REBT model of psychological health does a very good job of explaining participant's levels of posttraumatic stress symptoms.

In terms of the second objective, the results of the current analysis largely support initial predictions regarding the interrelations between the rational belief processes. Preference beliefs were found to positively predict all three secondary rational belief processes. Self-acceptance beliefs were the strongest predictor of posttraumatic stress symptoms recording a moderately-strong, negative, predictive effect. This result indicates that the more an individual internalises an accepting view of oneself the less likely that individual is to react negatively to traumatic life events. Current results support previous findings (Hyland et al. 2014c) highlighting the importance of self-acceptance beliefs in preventing posttraumatic stress responses. Acceptance beliefs as conceptualised in REBT theory share much in common with the types of beliefs and mind-sets that are advocated in the mindfulness-based interventions such as Acceptance and Commitment Therapy (Hayes 2005), Mindfulness-Based Stress 
Reduction (Kabat-Zinn 1990), and Mindfulness-Based Cognitive Therapy (Felder et al. 2012). While these mindfulness-based approaches stress the importance of acceptance as a key cognitive feature in prevention and recovery from psychopathology, acceptance beliefs in REBT are formulated somewhat differently (see David et al. 2013, for a fuller discussion). According to REBT theory, acceptance beliefs can take on two major forms: (i) a rational primary appraisal mechanism (e.g., preferences "I want to get it, but I can accept that it might not happen") and (ii) a rational secondary appraisal mechanism (e.g., unconditional acceptance of self, others, and/or life). Acceptance beliefs in REBT consequently reflect a more active, judgmental cognitive process than as outlined in the mindfulness-based approaches (Dryden and David 2008). Current results do suggest however that selfacceptance is an important factor in the prevention and/or alleviation of posttraumatic stress responses, and future research efforts could therefore benefit from exploring whether levels of mindfulness can also serve as a cognitive protective factor against the development of posttraumatic stress symptoms.

High frustration tolerance beliefs were also found to negatively impact on levels of posttraumatic stress symptoms to a moderate degree. These results indicate that the more an individual is capable of cultivating a belief in their own resilience and a belief in their ability to withstand negative traumatic life events and to continue to function in a productive manner, the less affected they are by life's traumas. There has been substantial evidence accumulated in recent years that 'distress intolerance' beliefs (conceptually equivalent to low frustration tolerance beliefs as described by REBT theory) are critical dysfunctional cognitions in the prediction of PTSD symptoms (Marshall-Berenz et al. 2010; Vujanovic et al. 2011). Current results suggest that individuals suffering from PTSD symptoms would benefit not only from reducing levels of distress intolerance/low frustration tolerance beliefs, but also from developing greater levels of high frustration tolerance beliefs. These findings lend credence to the REBT therapeutic approach to treating PTSD.

An interesting finding that was inconsistent with our initial hypothesis was that non-catastrophizing beliefs positively predicted levels of posttraumatic stress symptoms, albeit weakly. There are a number of plausible explanations for this finding. Traumatic life events are by their nature distressing and are almost universally evaluated as highly negative as indicated by research demonstrating that in the aftermath of a trauma a very high percentage of people report acute stress/ posttraumatic stress responses (Bryant et al. 2011). High levels of non-catastrophizing beliefs, as were observed in the current sample, are to be expected, even among a non-clinical sample. The discovery of a positive association between this belief process and posttraumatic stress symptoms are therefore not entirely surprising because even those who realistically evaluate the badness of a trauma would be expected to display some posttraumatic stress symptoms. Replication of the current analysis within a clinical sample is necessary to identify if the current finding is reliable or merely a result of utilizing a non-clinical sample. Clearly substantially greater research is required however based on previous findings (Hyland et al. 2014c) an interesting possibility exists that potentially one of the other rational belief processes may serve to moderate the impact of noncatastrophizing beliefs on levels of posttraumatic responses. In other words, 
although a weak, positive association may exist between non-catastrophizing beliefs and posttraumatic stress symptoms, if a person has a high level of acceptance or high frustration tolerance beliefs, these rational belief processes may serve to negate the effect of non-catastrophizing beliefs on posttraumatic stress responses.

Another explanation may relate to REBT's binary model of emotional distress (see DiLorenzo et al. 2011). The binary model of emotions predicts there is a qualitative distinction between functional and dysfunctional negative emotions; specifically, responding to a traumatic life event with a set of rational beliefs would be expected to give rise to functional negative symptoms. The PDS (Foa et al. 1997), used to capture posttraumatic stress symptoms, makes no accommodation for the distinction between functional and dysfunctional emotional responses, therefore it is possible that a proportion of the total distress captured by the PDS is comprised of functional negative emotions. It would be fully expected that non-catastrophizing evaluations of a serious traumatic life event would give rise to functional negative feelings. It is suggested therefore that the positive predictive effect of noncatastrophizing beliefs on posttraumatic stress symptoms may be an artefact of the PDS being unable to distinguish between functional and dysfunctional negative emotions.

In addition to the direct effects observed between each of the secondary rational beliefs and posttraumatic stress symptoms; two statistically significant, weak-tomoderate indirect effects were observed between preference beliefs and levels of posttraumatic stress symptomology via high frustration tolerance, and selfacceptance beliefs, respectively (see Table 3 ). These results provide empirical support for the predictions of REBT theory that the impact of preference beliefs on psychopathological symptoms is mediated via the secondary rational belief processes. Clearly the cross-sectional nature of the study design precludes the ability to determine mediating effects however the observation of these indirect relationships is supportive of such a relationship. Future studies will ideally employ longitudinal research designs to more reliably test this core prediction of REBT theory. The results of the SEM analysis do however provide the first piece of empirical evidence in support of the predictions of REBT theory regarding the organisation of the rational belief processes within its model of psychological health (David et al. 2010). Preference beliefs appear to be a primary rational appraisal mechanism while non-catastrophizing beliefs, high frustration tolerance beliefs, and acceptance beliefs appear to be secondary rational appraisal mechanisms.

The current study contains a number of limitations which ought to be considered by the reader. The most salient limitation of the current study relates to the attempt to test predictions of mediation with the use of cross-sectional data. Given that the current study was correlational in nature it was impossible to ascertain whether noncatastrophizing beliefs, high frustration tolerance beliefs, and acceptance beliefs actually mediate the relationship between preference beliefs and posttraumatic stress symptomology due to the temporal assumptions inherent in determining causality which mediation implies. Additionally, a self-report measure of posttraumatic stress symptoms was employed and although the PDS has been shown to highly correspond with clinician-administered measures (Griffin et al. 2004), clinician-based measures would have been preferable as they are considered the 
gold standard method of assessing posttraumatic stress symptomology. A further limitations associated with the current study that was impossible to avoid is that the self-report measure of posttraumatic stress responses used in this study was constructured to capture the symptoms of PTSD as outlined in the DSM-IV (APA 1994), and therefore does not measure the newly introduced symptoms of PTSD outlined in the recently published DSM-5 (APA 2013). Finally the introduction of a post hoc modification in the form of a correlated error between two of the indicators of posttraumatic stress symptoms is a major limitation of the current study and suggests that future studies investigating the effect of rational beliefs on symtopms of posttraumatic stress responses should ideally control for emotional distress; or more preferably that replication studies be performed among clinical samples.

In conclusion, the current study substantially contributes to the REBT literature and the wider trauma literature in a number of important ways. The results of the current analysis provide inital support for the REBT model of psychological health, the organisation of the rational beliefs, and the role of preference beliefs, high frustration tolerance beliefs, and acceptance beliefs in the allieviation of posttraumatic stress symptoms. These results have important clinical implications as clinicans can focus on developing and strengthening these functional cognitions in addition to challenging and disputing the more traditionally focused on dysfunctional cognitions, in their efforts to allieviate deleterious posttraumatic stress symptoms. The results of the current study will ideally stimulate further research to investigate the role of rational beliefs in the prevention of other psychiatric and psychological disorders, as well as investigating the predictions of REBT's theory of psychological health within positive mental health states such as happiness, optimism, resilience, and empathy, to name but a few.

\section{References}

American Psychiatric Association. (1994). Diagnostic and statistical manual of mental disorders (4th ed.). Washington, DC: Author.

American Psychiatric Association. (2013). Diagnostic and statistical manual of mental disorders (5th ed.). Washington, DC: Author.

Bentler, P. M. (1990). Comparative fit indices in structural models. Psychological Bulletin, 107, 238-246.

Bernard, M. E. (1998). Validation of the general attitudes and belief scale. Journal of Rational-Emotive \& Cognitive-Behavior Therapy, 16, 183-196.

Blake, D. D., Weathers, F. W., Nagy, L. M., Kaloupek, G. D., Charney, D. S., \& Keane, T. M. (1998). The Clinican-Administered PTSD Scale for DSM-IV. Boston: National Centre for PTSD, Behavioural Science Division.

Bollen, K. A. (1989). Structural equations with latent variables. New York: Wiley.

Bryant, R. A., Friedman, M. J., Spiegel, D., Ursano, R., \& Strain, J. (2011). A review of acute stress disorder in DSM-5. Depression and Anxiety, 28, 802-817.

Clark, D. A., \& Beck, A. T. (2010). Cognitive therapy of anxiety disorders. London: The Guilford Press.

David, D., Cote, C. D., Szentagotai, A., McMahon, J., \& DiGiuseppe, R. (2013). Philosophical versus psychological unconditional acceptance: Implications for constructing the Unconditional Acceptance Questionnaire. Journal of Cognitive and Behavioral Psychotherapies, 13, 445-464.

David, D., Lynn, S. J., \& Ellis, A. (2010). Rational and irrational beliefs: Research, theory, and clinical practice. Oxford: Oxford University Press. 
David, D., Schnur, J., \& Belloiu, A. (2002). Another search for the "hot" cognitions: Appraisal, irrational beliefs, attributions, and their relation to emotion. Journal of Rational-Emotive \& CognitiveBehavior Therapy, 15, 93-131.

David, D., \& Szentagotai, A. (2006). Cognition in cognitive-behavioral psychotherapies (CBT): Toward an integrative model. Clinical Psychology Review, 3, 284-298.

DiGiuseppe, R., Leaf, R., Exner, T., \& Robin, M.V. (1988). The development of a measure of rational/ irrational thinking. Paper presented at the World Congress of Behavior Therapy, Edinburgh, Scotland.

DiLorenzo, T. A., David, D., \& Montgomery, G. H. (2007). The interrelations between irrational cognitive processes and distress in stressful academic settings. Personality and Individual Differences, 42, 765-777.

DiLorenzo, T. A., David, D., \& Montgomery, G. H. (2011). The impact of general and specific rational and irrational beliefs on exam distress: A further investigation of the binary model of emotional distress as an emotional regulation model. Journal of Cognitive and Behavioural Psychotherapies, $11,121-142$.

Dryden, W., \& David, D. (2008). Rational emotive behavior therapy: Current status. Journal of Cognitive Psychotherapy: An International Quarterly, 22, 195-209.

Ehlers, A., \& Clark, D. M. (2000). A cognitive model of persistent posttraumatic stress disorder. Behaviour Research and Therapy, 38, 319-345.

Ellis, A., \& Dryden, W. (2007). The practice of rational emotive behaviour therapy (2nd ed.). New York: Springer Publishing Company.

Felder, J. N., Dimidjian, S., \& Segal, Z. (2012). Collaboration in mindfulness-based cognitive therapy. Journal of Clinical Psychology, 68, 179-186.

Foa, E., Cashman, L., Jaycox, L., \& Perry, K. (1997). The validation of a self-report measure of PTSD: The posttraumatic diagnostic scale. Psychological Assessment, 9, 445-451.

Foa, E. B., Ehlers, A., Clark, D. M., Tolin, D. F., \& Orsillo, S. M. (1999). The posttraumatic cognitions inventory (PTCI): Development and validation. Psychological Assessment, 11, 303-314.

Fulop, I. E. (2007). A confirmatory factor analysis of the attitude and belief scale 2. Journal of Cognitive and Behavioral Psychotherapies, 7, 159-170.

Griffin, M. G., Uhlmansiek, M. H., Resick, P. A., \& Mechanic, M. B. (2004). Comparison of the posttraumatic diagnostic scale versus the clinician-administered posttraumatic stress disorder scale in domestic violence survivors. Journal of Traumatic Stress, 17, 497-503.

Hair, J. F, Jr, Anderson, R. E., Tatham, R. L., \& Black, W. C. (1998). Multivariate data analysis with readings (5th ed.). Prentice Hall: New Jersey.

Hayes, S. C. (2005). Stability and change in cognitive behavior therapy: Considering the implications of ACT and REBT. Journal of Rational-Emotive and Cognitive-Behavior Therapy, 23, 131-151.

Hyland, P., \& Boduszek, D. (2012). Resolving a difference between cognitive therapy and rational emotive behaviour therapy: Towards the development of an integrated CBT model of psychopathology. Mental Health Review Journal, 17, 104-117.

Hyland, P., Shevlin, M., Adamson, G., \& Boduszek, D. (2013). The role of trauma- specific irrational beliefs and sociodemographic risk factors in posttraumatic stress responses. Journal of RationalEmotive \& Cognitive-Behaviour Therapy, 31, 152-156.

Hyland, P., Shevlin, M., Adamson, G., \& Boduszek, D. (2014a). The organisation of irrational beliefs in posttraumatic stress symptomology: Testing the predictions of REBT theory using structural equation modelling. Journal of Clinical Psychology, 70, 48-59. doi:10.1002/jclp.22009.

Hyland, P., Shevlin, M., Adamson, G., \& Boduszek, D. (2014b). Modelling the factor structure of the Attitudes and Belief Scale 2: Toward the development of an abbreviated version. Cognitive Behaviour Therapy, 43, 60-71.

Hyland, P., Shevlin, M., Adamson, G., \& Boduszek, D. (2014c). The moderating role of rational beliefs in the relationship between irrational beliefs and posttraumatic stress symptomology. Behavioural and Cognitive Psychotherapy, 42, 312-326.

Hyland, P., Shevlin, M., Adamson, G., \& Boduszek, D. (in press). Irrational beliefs in posttraumatic stress responses: A rational emotive behaviour therapy approach. Journal of Loss and Trauma: International Perspectives on Stress \& Coping. doi:10.1080/15325024.2013.839772.

Joreskog, K., \& Sorbom, D. (1981). LISREL V: Analysis of linear structural relationships by the method of maximum likelihood. Chicago: National Educational Resources.

Kabat-Zinn, J. (1990). Full catastrophe living. New York: Delacorte. 
King, D., Leskin, G., King, L., \& Weathers, F. (1998). Confirmatory factor analysis of the clinician administered PTSD Scale: Evidence for the dimensionality of posttraumatic stress disorder. Psychological Assessment, 10, 90-96.

Kline, R. B. (2005). Principles and practice of structural equation modeling (2nd ed.). London: The Guilford Press.

Macavei, B. (2002). Scala de Atitudini si Convingeri II (ABS II): Date preliminare pentru populatia de limba româna. Romanian Journal of Cognitive and Behavioral Psychotherapies, 2, 105-122.

MacCallum, R. C., \& Austin, J. T. (2000). Applications of structural equation modeling in psychological research. Annual Review of Psychology, 51, 201-226.

Marshall-Berenz, E. C., Vujanovic, A. A., Bonn-Miller, M. O., Bernstein, A., \& Zvolensky, M. J. (2010). Multi-method study of distress tolerance and PTSD symptom severity in a trauma-exposed community sample. Journal of Traumatic Stress, 23, 623-630.

Muthen, L. K., \& Muthen, B. O. (2012). Mplus: Statistical analysis with latent variables. User's guide (7th ed.). Muthen and Muthen: Los Angeles.

Pallant, J. (2007). SPSS survival manual (4th ed.). McGraw Hill: London.

Resick, P. A., \& Schnicke, M. K. (1993). Cognitive processing therapy for rape victims: A treatment manual. Newbury Park: Sage.

Resick, P. A., Schnicke, M. K., \& Markway, B. G. (1991, November). The relationship between cognitive content and PTSD. Paper presented at the 25th annual convention of the association for the advancement of behaviour therapy; New York, NY.

Simms, L. J., Watson, D., \& Doebbeling, B. N. (2002). Confirmatory factor analyses of posttraumatic stress symptoms in deployed and nondeployed veterans of the Gulf war. Journal of Abnormal Psychology, 111, 637-647.

Steiger, J. H. (1990). Structural model evaluation and modification: An interval estimation approach. Multivariate Behavioural Research, 25, 173-180.

Tiba, A. (2003). Rational and irrational beliefs from a neuroscience framework. Romanian Journal of Cognitive and Behavioral Psychotherapies, 3, 61-78.

Tucker, L. R., \& Lewis, C. (1973). The reliability coefficient for maximum likelihood factor analysis. Psychometrika, 38, 1-10.

Vogt, D. S., Shipherd, J. C., \& Resick, P. A. (2012). Posttraumatic maladaptive beliefs scale: Evolution of the personal beliefs and reactions scale. Assessment, 9, 308-317.

Vujanovic, A. A., Bonn-Miller, M. O., Potter, C. M., Marshall-Berenz, E. C., \& Zvolensky, M. J. (2011). An evaluation of the association between distress tolerance and posttraumatic stress within a traumaexposed sample. Journal of Psychopathology and Behavioral Assessment, 33, 129-135.

Yufik, T., \& Simms, L. J. (2010). A meta-analytic investigation of the structure of posttraumatic stress disorder symptoms. Journal of Abnormal Psychology, 119, 764-776. 